\title{
Assessing the viability of 3D printed brain models derived from MRI scan data in the communication of complex patterns of hypoxic ischaemic injury to lay-people
}

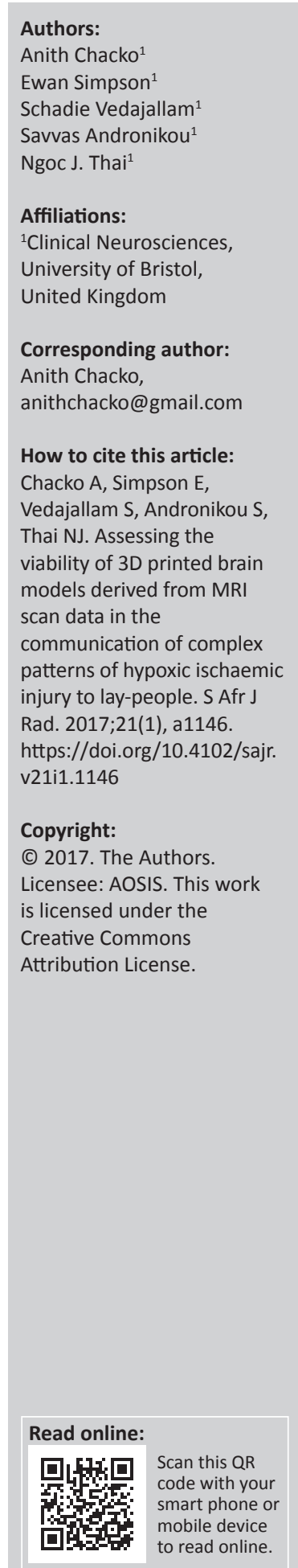

Background: Communication of bilateral, symmetric, zonal cortical brain atrophy in children with term hypoxic ischaemic injury (HII) to parents and the legal fraternity contesting compensation rights, using text reports, can be very difficult. 3D printed models, which may be viewed through grasping and rotating, may be useful for conveying such changes to non-radiologists and lay-people. In this report we describe the technical aspects of generating a 3D printed model from a paediatric brain MRI and provide completed examples in children with partial prolonged HII and controls.

Aim: To describe the technical aspects of producing 3D printed models of the brain in children using examples of children with term partial prolonged HII as well as age matched controls and to provide completed models for public consideration.

Methods and materials: The technical method for utilising 3DT1/3D FLAIR images, stripping the skull and converting to STereoLithography (STL) format is described as well as the 3D printing consideration for creating 3D models of the brain. MRI scans of 4 children, who demonstrated atrophy after having sustained partial prolonged HII at term and age matched controls were 3D printed using the described technique. These scans are used for demonstration of the advantages.

Results and conclusion:3D printing, using the described technique, was successful and generated 3D models in patients which could be compared with controls in contour and size for the purposes of diagnosing cortical atrophy in children who sustained peri-natal partial prolonged HII at term.

Note: A selection of conference abstracts: RSSA/SASPI Paediatric Imaging Congress, 03-06 November 2016, Spier Estate, Stellenbosch, South Africa. Faculty collaborators: Professor Kassa Darge (Body Imaging University of Pennsylvania, Philadelphia, USA), Professor South Africa. Faculty collaborators: Professor Kassa Darge (Body Imaging, University of Pennsylvania, Philadelphia, USA), Professo Edward Lee (Thoracic Imaging, Harvard University, USA), Professor Beverley Newman (Cardiac Imaging, Stanford University, California, USA), Professor Kimberly Applegate (Image Gently and Body lmaging, Emory University, Atlanta, USA) and Professor Savvas Andronikou (Thoracic Imaging, University of Bristol, UK) supported by South African Paediatric Radiologists, co-ordinated by Dr Jaishree Naidoo, President of the African Society of Paediatric Imaging and Head of Division of Paediatric Radiology, Charlotte Maxeke Johannesburg Academic Hospital. 